\title{
Die italienische Ratspräsidentschaft 2014: Ambitionen und Schwerpunkte unter schwierigen Bedingungen
}

\author{
Gianni Bonvicini und Ferdinando Nelli Feroci*
}

In der zweiten Hälfte des Jahres 2014 wird Italien, gemäß einem lange bestehenden Zeitplan, die Präsidentschaft des Rates der Europäischen Union übernehmen. Die Verantwortung der halbjährlichen Ratspräsidentschaft wurde im Rahmen der Reformen des Lissabon-Vertrags weitgehend beschnitten: Es wurden einige Neuerungen eingeführt, die der starken Fragmentierung des Arbeitsprogramms der Union entgegenwirken sollen. Beispiele sind die Einführung des permanenten Präsidenten des Europäischen Rates und die Stärkung der Rolle der Hohen Vertreterin der Union für Außen- und Sicherheitspolitik, die jetzt zugleich Vorsitzende des Außenministerrates ist. Dennoch bietet die Ratspräsidentschaft den Mitgliedstaaten Gelegenheit, die Aufmerksamkeit der Regierung, der Verwaltung und der Öffentlichkeit auf die europäische Politik zu lenken und eine gemeinsame Vision zur Durchsetzung der Ziele Europas sowie nationaler Interessen im europäischen Rahmen zu entwickeln.

Die Ratspräsidentschaft stellt außerdem einen Anreiz für das nationale politische System dar, seine Ausrichtung hin auf die Europäische Union zu optimieren und in Bezug auf verschiedene Aspekte der Mitgliedschaft Italiens in der Europäischen Union zu verbessern, etwa eine bessere Nutzung der Strukturfonds, einen besseren Zugang zu europäischen Fördermitteln für Forschung und Entwicklung, eine Reduzierung der Zahl der Vertragsverletzungsverfahren, eine Beschleunigung der Umsetzung europäischer Rechtsvorschriften in nationales Recht etc.

Bezüglich der internen Strukturen der italienischen Präsidentschaft gilt es zu berücksichtigen, dass sich in Italien erst kürzlich eine neue Regierung gebildet hat. Es ist daher schwierig, fundierte Empfehlungen bezüglich der Rollenverteilung und Verantwortung der verschiedenen Protagonisten in der Regierung im Hinblick auf das Management der Ratspräsidentschaft zu formulieren. Entsprechend einem Trend, der sich in den letzten Jahren gefestigt hat, ist es jedoch sehr wahrscheinlich, dass die Verantwortung für maßgebliche Impulse und für die politische Koordinierung der verschiedenen Initiativen vornehmlich beim Ministerpräsidenten und der Ministerpräsidentschaft liegen wird. In der Ausübung dieser Verantwortung wird der Ministerpräsident durch einen Staatssekretär (diesmal direkt bei der Ministerpräsidentschaft angesiedelt) unterstützt, der die Funktionen und Aufgabenbereiche des bisherigen Ministers für europäische Angelegenheiten übernimmt. Die Minister für Auswärtige Angelegenheiten sowie für Wirtschaft und Finanzen werden in ihren jeweiligen Zuständigkeitsbereichen eine wichtige Rolle bei der Unterstützung und Durchführung der Maßnahmen des Ministerpräsidenten spielen. Sowohl der Ministerpräsident als auch der Staatssekretär für europäische Angelegenheiten werden sich im Zusammenhang mit den Aufgaben der Ratspräsidentschaft weiterhin der Strukturen und Expertise des Außenministeriums bedienen.

* Prof. Dr. Gianni Bonvicini, Vizepräsident des Istituto Affari Internazionali, Rom. Prof. Dr. Ferdinando Nelli Feroci, Präsident des Istituto Affari Internazionali, Rom. 


\section{Chancen und Herausforderungen}

Zunächst ist darauf hinzuweisen, dass das laufende Jahr 2014 große institutionelle Veränderungen in der Union mit sich bringt. In den Tagen vom 22. bis 25. Mai 2014 wurde ein neues Europäisches Parlament gewählt, das frühestens im September arbeitsfähig sein wird. In den ersten Monaten nach der Wahl werden die politischen Folgen des Wahlergebnisses, die Zusammensetzung der Fraktionen und die Wahl der neuen Führungskräfte (Präsident des Europäischen Parlaments und Vorsitzende der parlamentarischen Ausschüsse) im Zentrum der Aufmerksamkeit stehen.

Im Oktober 2014 endet das Mandat der Europäischen Kommission. Das Verfahren der Berufung der neuen Kommission beginnt voraussichtlich bereits im Juni 2014 mit der Ernennung des Präsidenten, der vom Europäischen Rat vorgeschlagen und vom neuen Parlament gewählt wird. Darauf folgt die Ernennung des Vizepräsidenten der Kommission/Hohen Vertreters sowie der weiteren Kommissionsmitglieder, die vom Rat im Einvernehmen mit dem Kommissionspräsidenten vorgeschlagen werden und der Bestätigung durch das Parlament bedürfen. Schließlich läuft Ende November 2014 auch das Mandat des Präsidenten des Europäischen Rates aus. Der neue Amtsinhaber wird voraussichtlich ebenfalls bereits im Juni 2014 als Teil eines ausgewogenen ,Nominierungspakets‘ ernannt werden.

Die Termine der Neubesetzung der europäischen Spitzenpositionen werden sich stark auf die italienische Ratspräsidentschaft auswirken. So wird sich Italien nicht in dem Maße auf die Zusammenarbeit mit der Europäischen Kommission verlassen können, wie es sonst bei Ratspräsidentschaften der Fall ist, und es wird sich sowohl auf eine längere Arbeitspause des Parlaments als auch den Wechsel im Amt des Präsidenten des Europäischen Rates einstellen müssen.

Italien kommt somit die schwierige Aufgabe zu, die Kontinuität der europäischen Politik in einer komplexen Übergangsphase zu sichern. Es ist mit dafür verantwortlich, dass die verschiedenen Schritte auf dem Weg zur Erneuerung der Spitzenposten konsensorientiert von statten gehen. Dabei gilt es insbesondere, inter-institutionellen Konflikten vorzubeugen.

Italien sollte diese Phase institutioneller Umwälzungen durch eine Strategie vorbereiten, die sich effektiv auf die Termine und Fristen der europäischen Programme konzentriert. Ziel sollte es sein, durch engagierte Abgeordnete im kommenden Europäischen Parlament vertreten zu sein, die den gestärkten Kompetenzen des Europäischen Parlaments und der damit verbundenen gewachsenen Verantwortung gerecht werden. Außerdem sollte Italien sich aktiv an der Wahl des zukünftigen Kommissionspräsidenten beteiligen sowie an den Konsultationen mit dem Parlament, die der Ernennung seitens des Europäischen Rates vorausgehen. Ebenso sollte es aktiv an der Wahl des zukünftigen Präsidenten des Europäischen Rates und des Hohen Vertreters mitwirken. Falls es nicht gelingt, einen der Spitzenposten mit einem italienischen Kandidaten zu besetzen, sollte das italienische Kommissionsmitglied mit Sorgfalt ausgewählt werden - auch im Hinblick auf die Besetzung eines Ressorts, das den Interessen Italiens entspricht.

Darüber hinaus agiert die italienische Präsidentschaft in einem Umfeld, das Anfang 2014 dadurch gekennzeichnet bleibt, dass die Aussichten und der Umfang der wirtschaftlichen Erholung unsicher bleiben. Es fehlt an Vertrauen in die Fähigkeit der Union, die in den letzten Jahren - im Vergleich zu globalen Partnern - stark geschwächte Wettbewerbsfähigkeit entscheidend wiederherzustellen. Weiterhin bedarf es einer Debatte über die Fortentwicklung des gemeinsamen europäischen Projekts. Im Übrigen schwindet das Vertrauen der Öffentlichkeit in die europäischen Institutionen, die (zu Recht oder zu Unrecht) für eine Politik 
verantwortlich gemacht werden, die Wachstum und Beschäftigung auf dem Altar der Austerität geopfert hat.

Zusätzlich zu dieser ohnehin schwierigen Situation, sind die Beziehungen zwischen den Mitgliedstaaten durch einen fortwährenden Mangel an Vertrauen gekennzeichnet, der durch die Folgen der Wirtschaftskrise noch verschärft wurde. Die Aufteilung der Verantwortlichkeiten zwischen den nationalen Regierungen und den EU-Institutionen ist diffus. Es herrschen latente Spannungen zwischen Mitgliedern und Nicht-Mitgliedern der Eurozone. Hinzu kommen Bedenken wegen der für 2014 geplanten Referenden über die Unabhängigkeit Schottlands und Kataloniens. Darüber hinaus bergen mögliche Forderungen Großbritanniens, die Bedingungen seiner Mitgliedschaft neu zu verhandeln, neues Konfliktpotenzial.

Die italienische Präsidentschaft wird nicht umhin kommen, die genannten Eckdaten in Betracht zu ziehen. Gleichzeitig muss die Ratspräsidentschaft aber auch darauf hinwirken, die Bedingungen für die politische Unterstützung des europäischen Projekts wiederherzustellen. Zusammen mit weiteren Regierungen, die sich im europäischen Rahmen besonders engagieren, muss die italienische Präsidentschaft eine starke politische Initiative ergreifen, mit dem primären Ziel, die euroskeptischen Kräfte einzudämmen. Diese politische Initiative sollte aber auch dem längerfristigen Ziel einer ehrgeizigen Wiederbelebung des Integrationsprojekts Rechnung tragen.

Bezüglich der einzelnen Dossiers wird einiges davon abhängen, wie viel die italienische Ratspräsidentschaft vom Arbeitsprogramm der vorausgehenden griechischen Präsidentschaft ${ }^{1}$ übernimmt und wie viele legislative Verfahren das Parlament nach den Wahlen im Mai 2014 wieder aufnehmen wird. Dennoch ist es bereits möglich, einige Punkte zu formulieren, die in das Arbeitsprogramm der italienischen Präsidentschaft aufgenommen werden müssen.

\section{Die wirtschaftspolitische Steuerung in der Europäischen Union stärken und eine Politik des Wachstums und der Beschäftigung fördern}

Die Vervollständigung der Bankenunion und die Reform der wirtschaftspolitischen Steuerung werden weiterhin im Mittelpunkt der europäischen Agenda stehen. Hinsichtlich der Bankenunion wurde die Verordnung zum ,einheitlichen Überwachungsmechanismus“2 verabschiedet. Im Gesetzgebungsverfahren zur Verordnung, die das Verfahren für die ,Bankenabwicklung ' festlegt, wurde ein Kompromiss zwischen Europäischem Parlament und Rat erreicht. ${ }^{3}$ Die genannten Verordnungen werden gemäß den jeweiligen Übergangsfristen in

1 Programme of the Hellenic Presidency of the Council of the European Union, 1 January - 30 June 2014. Europe: Our Common Quest, abrufbar unter: http:/gr2014.eu/sites/default/files/PROGRAMME\%28EN\%2928012014. pdf (letzter Zugriff: 31.3.2014).

2 Verordnung (EU) Nr. 1024/2013 des Rates vom 15. Oktober 2013 zur Übertragung besonderer Aufgaben im Zusammenhang mit der Aufsicht über Kreditinstitute auf die Europäische Zentralbank, in: Amtsblatt der EU, Nr. L 287 vom 29. Oktober 2013, S. 63-89; Verordnung (EU) Nr. 1022/2013 des Europäischen Parlaments und des Rates vom 22. Oktober 2013 zur Änderung der Verordnung (EU) Nr. 1093/2010 zur Errichtung einer Europäischen Aufsichtsbehörde (Europäische Bankenaufsichtsbehörde) hinsichtlich der Übertragung besonderer Aufgaben auf die Europäische Zentralbank gemäß der Verordnung (EU) Nr. 1024/2013, in: Amtsblatt der EU, Nr. L 287 vom 29. Oktober 2013, S. 5-14.

3 Europäisches Parlament, Wirtschaft und Währung: Parliament negotiators rescue seriously damaged bank resolution system, Pressemitteilung, 20.3.2014; Europäische Kommission: Vorschlag für eine Verordnung des Europäischen Parlaments und des Rates zur Festlegung einheitlicher Vorschriften und eines einheitlichen Verfahrens für die Abwicklung von Kreditinstituten und bestimmten Wertpapierfirmen im Rahmen eines einheitlichen Abwicklungsmechanismus und eines einheitlichen Bankenabwicklungsfonds sowie zur Änderung der Verordnung (EU) Nr. 1093/2010 des Europäischen Parlaments und des Rates, KOM (2013) 520. 
Kraft treten. Der einheitliche Abwicklungsmechanismus war bislang Gegenstand eines schwierigen und kontroversen politischen Übereinkommens innerhalb des Rates sowie zwischen Rat und Europäischem Parlament. Er konnte nunmehr im April 2014 beschlossen werden. 2014 ist jedoch vor allem das Jahr, in dem die Europäische Zentralbank die Qualität der Aktiva der Banken prüfen muss, ${ }^{4}$ die der zentralen Aufsicht unterstellt sind. Außerdem wird die Europäische Bankenaufsichtsbehörde diese Banken einer weiteren Runde von Stresstests unterziehen. ${ }^{5}$ Dies sind zwei heikle Schritte, die als Grundlage für die Aufnahme der Arbeit des Aufsichtsmechanismus der Europäischen Zentralbank gelten. Diese ist für den kommenden September 2014 geplant. Im Frühjahr und nach dem Sommer 2014 gilt es daher sicherzustellen, dass die Bankenunion, und somit ein entscheidender Teil der Reform der wirtschaftspolitischen Steuerung, so effektiv und glaubwürdig wie möglich umgesetzt wird. Dies kann dabei helfen, die Zersplitterung der europäischen Finanzmärkte in der Eurozone zu beheben oder zumindest zu reduzieren. Die Umsetzung der Bankenunion kann auch dazu beitragen, den Teufelskreis zwischen Länderrisiken und Risiken des Bankensystems zu brechen und den Markt der Kreditvergabe für Unternehmen und Privathaushalte insbesondere in den Peripherieländern - wiederzubeleben.

In den kommenden Monaten bis zum Europäischen Rat im Oktober 2014 wird die Reform der wirtschaftspolitischen Steuerung insbesondere durch die Definition von vertraglichen Vereinbarungen ${ }^{6}$ für Wachstum und Beschäftigung fortgeführt. Diese Maßnahme dient einer Optimierung der Koordinierung nationaler Wirtschaftspolitiken, mit dem Ziel Wettbewerbsfähigkeit, Wachstum und Beschäftigung zu steigern. Die italienische Regierung sollte eigene Vorschläge zu diesen vertraglichen Vereinbarungen beisteuern und sicherstellen, dass das neue Instrument ohne Diskriminierung auf alle Mitglieder der Eurozone angewandt wird. Außerdem obliegt es ihr, den Prioritäten der einzelnen Mitgliedstaaten Rechnung zu tragen und sicherzustellen, dass das neue Instrument über ausreichend demokratische Legitimität verfügt und durch glaubwürdige Solidaritätsmechanismen abgerundet wird.

Schließlich sollte man versuchen, ein Arbeitsprogramm zu entwerfen, das Wachstum und Beschäftigung auf glaubwürdige Weise wieder in den Mittelpunkt der europäischen Agenda stellt. In erster Linie geht es um eine weitreichende politische Aktion, die die Regierungen der Mitgliedstaaten und die EU-Institutionen von der Dringlichkeit einer Abkehr von ausschließlich auf die Kontrolle öffentlicher Haushalte abzielender Politik überzeugt. Diese Initiative sollte die bereits beschlossenen Maßnahmen bezüglich der Haushaltsdefizite und der öffentlichen Verschuldung nicht infrage stellen. Mit Blick auf die Nachhaltigkeit der öffentlichen Finanzen sollte sie jedoch die Voraussetzungen für ein gewisses Maß an Flexibilität schaffen, zumindest was die zeitlichen Vorgaben für die Erreichung der vereinbarten Ziele betrifft.

Der gemeinsame europäische Haushalt sieht keine Mittel für antizyklische Politik vor. Eine Ausnahme bilden die Strukturfonds, die lange Programmlaufzeiten haben und restrik-

4 Europäische Zentralbank: Mitteilung. Umfassende Bewertung, Oktober 2013, abrufbar unter: http://www.ecb. europa.eu/pub/pdf/other/notecomprehensiveassessment201310de.pdf (letzter Zugriff: 5.5.2014).

5 Ebenda.

6 Europäische Kommission: Mitteilung der Kommission an das Europäische Parlament und den Rat. Auf dem Weg zu einer vertieften und echten Wirtschafts- und Währungsunion. Einführung eines Instruments für Konvergenz und Wettbewerbsfähigkeit, KOM (2013) 165. 
tiven Bedingungen unterliegen, sowie die Mittel aus der Fazilität „Connecting Europe“, 7 die der Finanzierung großer transeuropäischer Infrastrukturprojekte dienen. Daher müssen sich spezifische Maßnahmen für eine europäische Wachstumsagenda weiterhin auf die Rahmenbedingungen konzentrieren. Der Binnenmarkt - auch für Dienstleistungen - sollte also vervollständigt werden. Des Weiteren sollten die laufenden Verhandlungen über Freihandelszonen (vor allem die transatlantische Handels- und Investitionspartnerschaft (TTIP) mit den Vereinigten Staaten) beschleunigt, die administrativen und bürokratischen Auflagen insbesondere für Unternehmen vereinfacht und die Umsetzung der Digitalen Agenda mit Nachdruck verfolgt werden. Schließlich sollte man neue Finanzierungsquellen für die Realwirtschaft erschließen und dabei das Potenzial der Europäischen Investitionsbank in Kombination mit den vorgesehenen Haushaltsinstrumenten der Europäischen Union nutzen.

\section{Die Prioritäten in der Außen- und Sicherheitspolitik}

Was die Außenpolitik der Union betrifft, sollte sich Italien zunächst auf die Definition langfristiger strategischer Prioritäten für die europäische Agenda konzentrieren. Dieser Weg wurde bereits teilweise von den Regierungen Monti und Letta beschritten. Doch nur in wenigen Fällen gelang es Italien bisher, eine führende Rolle bei der Definition der internationalen Agenda der Europäischen Union zu übernehmen. Ein Beispiel war Italiens Förderung eines aktiven europäischen Ansatzes für Sicherheit und Entwicklung in Somalia und am Horn von Afrika. Weitere Beispiele sind die Unterstützung einer einheitlichen europäischen Position zu Gunsten einer diplomatischen (und militärischen) Lösung des Bürgerkriegs in Syrien sowie die Einbeziehung des Irans in die multilateralen Verhandlungen der „Geneva II Middle East Peace Conference on Syria“.

Nur ein qualitativer Sprung von der bloßen Anwesenheit am Verhandlungstisch hin zu tatsächlichem Einfluss auf die europäische Außenpolitik wird es Italien ermöglichen, seine Interessen im Einklang mit der Unterstützung für die internationale Rolle der Europäischen Union zu verfolgen. ${ }^{8}$ Es bedarf allerdings auch größerer Bemühungen zur Verwirklichung dieser Ziele, ohne dass weder die verfügbaren Ressourcen noch die Stärkung der nationalen und europäischen Identität außer Acht gelassen werden.

Unserer Meinung nach sind die folgenden Prioritäten die entscheidenden:

Einen neuen europäischen Ansatz zur Nachbarschaft fördern: Konkrete Maßnahmen in diese Richtung umfassen die Stärkung der Partnerschaft zwischen der Europäischen Union und den wichtigsten Akteuren im erweiterten Mittelmeerraum von den arabischen Ländern Nordafrikas bis hin zur Golfregion. Die Stärkung dieser Partnerschaften kann sich auf Italiens Expertise in bestimmten Bereichen stützen, wie beispielsweise die Reform des libyschen Sicherheitssektors. 2.000 libysche Soldaten werden zukünftig von einem italienischen Kontingent von 100 Mann im Rahmen der italienischen Mission in Libyen geschult. Italien ist zudem eines der europäischen Länder, die sich besonders dazu eignen, die Beitrittsperspektiven der Länder des westlichen Balkans (insbesondere die Beitrittsverhandlungen mit Ser-

7 Verordnung (EU) Nr. 1316/2013 des Europäischen Parlaments und des Rates vom 11. Dezember 2013 zur Schaffung der Fazilität „Connecting Europe“, zur Änderung der Verordnung (EU) Nr. 913/2010 und zur Aufhebung der Verordnungen (EG) Nr. 680/2007 und (EG) Nr. 67/2010, in: Amtsblatt der EU, Nr. L 348 vom 20. Dezember 2013, S. 129-171.

8 Gianni Bonvicini et al.: L'Italia in un mondo che cambia. Suggerimenti per la politica estera italiana, Istituto Affari Internazionali: Ufficio Studi Finmeccanica, Rom, Juni 2012. 
bien) sowie möglicherweise auch der Türkei ${ }^{9}$ (mit speziellem Augenmerk auf die Eröffnung des Kapitels 24 zu Justiz und Innerem), konkret voranzubringen. Die östliche Dimension der Europäischen Nachbarschaft sollte auch besondere Aufmerksamkeit erhalten, vor allem in Hinblick auf einen gewichtigen Beitrag der Europäischen Union zur Lösung der Krise in der Ukraine. ${ }^{10}$

Die transatlantische Partnerschaft in einer multipolaren Welt stärken: Italien muss das Ziel eines Abschlusses des Freihandelsabkommens mit den Vereinigten Staaten weiterhin mit Nachdruck verfolgen. Das Abkommen könnte auch einen Anstoß zur Neujustierung der Außenpolitik der Vereinigten Staaten zugunsten Europas geben, vor dem Hintergrund einer zunehmenden amerikanischen Ausrichtung nach Asien. Darüber hinaus sollte Italien seine guten Beziehungen zur russischen Führung nutzen, um eine kohärentere und effizientere gemeinsame europäische Russlandpolitik zu fördern. Dies könnte auch zu einer besseren Koordinierung der Positionen der Vereinigten Staaten, Europas und Russlands bezüglich spezifischer Sicherheitsaspekte und des Managements größerer Krisenregionen - von Syrien bis hin zur Ukraine - beitragen.

Interessen und Prioritäten der strategischen Partnerschaften der Europäischen Union definieren: Das Instrument der strategischen Partnerschaften könnte eine treibende Kraft für den Erfolg der internationalen Rolle der Europäischen Union sein. Dafür müssen jedoch Ziele und Vorgehensweise fallspezifisch und unter Berücksichtigung der wichtigsten wirtschaftlichen und politischen Interessen der Europäischen Union festgelegt werden. Dies erfordert eine stärkere Koordinierung der Außenpolitiken der Mitgliedstaaten und die Definition gemeinsamer europäischer Positionen. Italien muss einen Beitrag leisten, um die ,Scheckbuchdiplomatie ' oder die Realpolitik gegenüber Ländern wie China ${ }^{11}$ und Russland einzudämmen und einen politischen Dialog zu Demokratie und Menschenrechten zu fördern. Der nächste entscheidende Termin dafür ist der ASEM-Gipfel ${ }^{12}$ in Mailand im Oktober 2014.

Stärkung der euro-afrikanischen Beziehungen: Sub-Sahara-Afrika ist die größte Herausforderung für Europa im 21. Jahrhundert. Um glaubwürdig zu sein, muss sich die Afrikapolitik der Europäischen Union, durch die Identifizierung gemeinsamer strategischer Prioritäten, vom post-kolonialen Erbe emanzipieren. Italien kann zum Fürsprecher einer gerechteren Verteilung von Ressourcen und europäischer Präsenz auf dem afrikanischen Kontinent in den Bereichen Sicherheit und Entwicklung werden. Es sollte sich insbesondere dafür einsetzen, dass die Aufmerksamkeit der Europäischen Union hinsichtlich der Stabilisierung Somalias groß bleibt und dass der regionale und multidimensionale Ansatz zur Sicherheit am Horn von Afrika weiter verfolgt wird.

Größere Kohärenz zwischen internen und externen Politiken der Europäischen Union fördern: Die Anerkennung der externen Dimension interner Politiken der Europäischen Union, unter denen Energie und Immigration die bedeutendsten sind, ist eine der wichtigsten Entwicklungen in der Strategieformulierung seit den Reformen des Lissabon-Vertrags. Italien muss diesen Ansatz weiter stärken, indem es zu externen Politiken der Europäischen Union beiträgt, die internen Primärinteressen - wie dem gemeinsamen Management des

9 Zum Prozess der Europäisierung der Türkei siehe Olaf Leiße/MartaTryk: Der Dauerkandidat. Die Europäisierung der Türkei unter der AKP, in: integration 1/2014, S. 45-64.

10 Zur Krise in der Ukraine siehe Katrin Böttger: Auf dem sicherheitspolitischen Auge blind: Die EU-Außenpolitik angesichts der Ukraine-Krise: Zustand und Entwicklungsoptionen, in: diesem Band, S. 85-108.

11 Zur europäischen Politik gegenüber China siehe Sebastian Bersick/Jörn-Carsten Gottwald: Von wegen Zivilmacht: 10 Jahre Strategische Partnerschaft der Europäischen Union und der Volksrepublik China, in: integration 4/2012, S. 291-307.

12 Asia-Europe Meeting. 
Migrationsproblems oder der Diversifizierung der Energiequellen - dienen. Italien kann auf die tragende Rolle seines zivilen und militärischen Personals in der Gemeinsamen Sicherheits- und Verteidigungspolitik verweisen, um die Debatte über die Verknüpfungen von innerer und äußerer Sicherheit und deren Auswirkungen auf die Außenpolitik der Europäischen Union zu intensivieren.

\section{Praktische Anforderungen im Bereich der Verteidigung im Hinblick auf den entsprechenden Europäischen Rat in $\mathbf{2 0 1 5}$}

Der Europäische Rat hat im Dezember 2013 die Mitgliedstaaten aufgefordert, „die Verteidigungszusammenarbeit zu vertiefen"13 - einschließlich operativer, budgetärer, technologischer, industrieller sowie umfassender strategischer Aspekte. Obwohl ein vollständiges Paket von Analysen und Vorschlägen vorlag, hat der Europäische Rat keine wichtigen Entscheidungen getroffen. Er hat jedoch die von der Hohen Vertreterin, der Europäischen Verteidigungsagentur und der Kommission erstellten Unterlagen positiv aufgenommen. ${ }^{14}$ In diesem Sinne wurden die Hohe Vertreterin, die Europäische Verteidigungsagentur und die Kommission beauftragt, eine Reihe von Maßnahmen zu erarbeiten. Darunter befinden sich verschiedene Dokumente und proaktive Umsetzungsfahrpläne mit Fristen zwischen Juni 2014 und Ende 2015, die die Annahme von Beschlüssen ermöglichen. Vieles wird hierbei von den Ambitionen und Fähigkeiten des (zukünftigen) Hohen Vertreters sowie von der Zusammenarbeit zwischen der Europäischen Verteidigungsagentur und der Kommission abhängen.

Italien sollte sich bereits im Vorfeld der Präsidentschaft Gedanken über die Fortführung des vom Europäischen Rat angestoßenen Prozesses machen. Hierbei gilt es, soweit wie möglich zu verhindern, dass die geforderten Maßnahmen sich in einem Teufelskreis erschöpfen, in dem Mitteilungen nur weitere Studien und Papiere anstoßen, ohne dass wirkliche Entscheidungen getroffen werden.

Alle Punkte des Abschlussdokuments des Europäischen Rates vom Dezember $2013^{15}$ sind wichtig und sollten weiterentwickelt werden. Dennoch erscheinen uns einige von besonderem Interesse für die italienische Ratspräsidentschaft:

Der Europäische Rat hat die Kommission und die Hohe Vertreterin beauftragt, bis Juni 2014 einen gemeinsamen Entwurf für eine Europäische Maritime Sicherheitsstrategie vorzulegen. ${ }^{16}$ Der Verabschiedung der Strategie folgt die Vorbereitung von Aktionsplänen zur Bewältigung maritimer Herausforderungen. ${ }^{17}$ Die Präsidentschaft sollte sich um die Verabschiedung dieser globalen Maritimen Sicherheitsstrategie kümmern und sich insbesondere dafür einsetzen, dass diese baldmöglichst durch Aktionspläne zu den verschiedenen politischen, strategischen, operativen und technologischen Aspekten ergänzt wird. Hierbei gibt es viele offene Fragen, einschließlich der Probleme bezüglich der Hoheitsgewässer, der aus-

13 Europäischer Rat: Europäischer Rat (Tagung vom 19./20. Dezember 2013). Schlussfolgerungen, Euco 217/13, S. 2.

14 Ebenda.

15 Ebenda.

16 Ebenda, S. 4.

17 Obwohl sich unter diesen sicherlich auch die Verteidigung maritimer Grenzen sowie der Umgang mit illegaler Migration befänden, sollen diese Probleme, dem Rat zufolge, im Rahmen der Gemeinsamen Sicherheits- und Verteidigungspolitik angegangen werden. Dies soll in zunehmender Synergie mit den Verantwortlichen für Freiheit, Sicherheit und Recht geschehen. Ziel ist es, ,horizontale‘ Kapazitäten zu schaffen, die nicht nur der Bekämpfung irregulärer Immigration, sondern auch der organisierten Kriminalität, des Terrorismus etc. dienen. 
schließlichen Wirtschaftszonen, der zahlreichen Konflikte zur Anwendung des Seerechts, der Migrationsströme, der Kriminalität etc.

Der Europäische Rat ist überzeugt, dass gesteigerte Transparenz und Informationsaustausch in der Verteidigungsplanung zu einer erhöhten Konvergenz von Kapazitätsbedürfnissen und Zeitrahmen führen. Zu diesem Zweck werden die Hohe Vertreterin und die Europäische Verteidigungsagentur bis Ende 2014 einen politischen Rahmen vorbereiten. Diese Maßnahme steht im Einklang mit der Politik der Liberalisierung und Vereinheitlichung des europäischen Verteidigungsmarkts, die Italien in den vergangenen Jahren verfolgt hat. Ziel der Ratspräsidentschaft sollte es sein, einen politischen Rahmen mit vorzubereiten und die Arbeiten daran - wo nötig - voranzutreiben. Sie sollte im Voraus darauf hinwirken, dass ein größtmöglicher Konsens im Rat zum Zeitpunkt der Präsentation der Vorschläge herbeigeführt worden ist.

Der Europäische Rat bekräftigte sein Interesse an der Erhaltung und Stärkung der europäischen rüstungstechnologischen und -industriellen Basis (EDTIB) und begrüßte die Analysen und Überlegungen der Kommission, die sie ihm in der Mitteilung „Auf dem Weg zu einem wettbewerbsfähigeren und effizienteren Verteidigungs- und Sicherheitssektor" ${ }^{\text {"18 }}$ vorlegte. ${ }^{19}$ Er unterstützt die Idee, dass die Kommission, zusammen mit der Hohen Vertreterin und der Europäischen Verteidigungsagentur, einen Umsetzungsfahrplan entwickelt. Dieser sollte in vollem Einklang mit den beiden verteidigungsspezifischen Richtlinien des Jahres $2009^{20}$ stehen und auf die Integration des europäischen Komponenten- und Zulieferermarkts abzielen. Da der Kommission für diese Aufgabe keine zeitliche Frist gestellt wurde, ist es Aufgabe der Präsidentschaft, die Arbeit der Institutionen zu befördern und den Prozess zu beschleunigen. In diesen Rahmen reiht sich der Vorschlag, den Zugang der kleinen und mittleren Unternehmen des Verteidigungssektors zum Binnenmarkt zu erleichtern und deren technologische Wettbewerbsfähigkeit zu fördern.

Der Europäische Rat hat die Europäische Verteidigungsagentur und die Kommission auch beauftragt, einen Fahrplan für die Entwicklung gemeinsamer europäischer Industriestandards für den Verteidigungssektor zu erarbeiten. Außerdem hat er die Europäische Verteidigungsagentur aufgefordert, Optionen zur Kostenreduktion für die militärische Zertifizierung zu entwickeln, einschließlich der gegenseitigen Anerkennung nationaler Zertifizierungen. ${ }^{21}$ Diese Maßnahmen werden sich voraussichtlich Mitte 2014 konkretisieren. Sie fallen somit der Ratspräsidentschaft zu, die sicherstellen sollte, dass sie ohne Verzögerung von entsprechenden Entscheidungs- und operativen Phasen durchgeführt werden.

Es ist essenziell, die Versorgungssicherheit zu gewährleisten. Das bedeutet, dass es keine politischen oder anderweitigen Gründe gibt, die einen Mitgliedstaat von der Akquise bei Unternehmen eines anderen Mitgliedstaats abhalten. Die Europäische Verteidigungsagentur

18 Europäische Kommission: Mitteilung der Kommission an das Europäische Parlament, den Rat, den Europäischen Wirtschafts- und Sozialausschuss und den Ausschuss der Regionen. Auf dem Weg zu einem wettbewerbsfähigeren und effizienteren Verteidigungs- und Sicherheitssektor, KOM (2013) 542.

19 Europäischer Rat: Schlussfolgerungen, 2013, S. 7-8.

20 Richtlinie 2009/43/EG des Europäischen Parlaments und des Rates vom 6. Mai 2009 zur Vereinfachung der Bedingungen für die innergemeinschaftliche Verbringung von Verteidigungsgütern, in: Amtsblatt der EU, Nr. L 146 vom 10. Juni 2009, S. 1-36; Richtlinie 2009/81/EG des Europäischen Parlaments und des Rates vom 13. Juli 2009 über die Koordinierung der Verfahren zur Vergabe bestimmter Bau-, Liefer- und Dienstleistungsaufträge in den Bereichen Verteidigung und Sicherheit und zur Änderung der Richtlinien 2004/17/EG und 2004/18/EG, in: Amtsblatt der EU, Nr. L 216 vom 20. August 2009, S. 76-136.

21 Europäischer Rat. Schlussfolgerungen, 2013, S. 9. 
hat vor Kurzem eine Rahmenübereinkunft zur Versorgungssicherheit ${ }^{22}$ verabschiedet. Außerdem hat der Europäische Rat die Kommission aufgefordert einen Fahrplan zu entwickeln, mit dem Ziel, ein umfassendes System der europäischen Versorgungssicherheit zu errichten. Es wird Aufgabe der Präsidentschaft sein, die von den Institutionen angestoßenen Maßnahmen voranzutreiben und den Weg für die anschließende Entscheidungsphase zu ebnen.

Viele der weiteren Punkte in den Schlussfolgerungen des Europäischen Rates sind von großer Bedeutung. Die oben aufgeführten scheinen jedoch bereits reif für eine baldige Umsetzung.

\section{Für eine effizientere europäische Migrationspolitik}

In den vergangenen Monaten wurden Migrationsthemen dramatisch aktuell. Dies war eine Folge tragischer Ereignisse, die das Leben hunderter von Menschen kosteten, welche versuchten, das Territorium der Europäischen Union zu erreichen. Allgemeiner gesagt wird das Problem einer gemeinschaftlichen Politik der Lenkung von Migrationsströmen in den kommenden Jahren eine der Prioritäten der europäischen Politik sein. Es sollten weitere Maßnahmen und Initiativen im Bereich der Migrationspolitik beschlossen werden, mit dem Ziel, das Solidaritätsprinzip besser umzusetzen und sowohl die Aufnahme von Migranten als auch die Kontrolle an den Außengrenzen der Union effizienter zu gestalten. Derweil wird ein neues Mehrjahresprogramm ${ }^{23}$ im Bereich der Innenpolitik und der Justiz beschlossen, das das 2014 auslaufende Stockholmer Programm ${ }^{24}$ ablöst. $^{25}$

Auf europäischer Ebene fordert Italien eine stärkere Rolle der supranationalen Institutionen und eine bessere Verteilung der Kosten, die ihre Rechtfertigung in der Rolle Italiens als Wächter der gemeinsamen Außengrenze finden. Trotz eines soliden überparteilichen Konsenses, gibt es hierzu bisher nur Grundsatzerklärungen. Es ist nicht gelungen, diese Forderungen in eine Verhandlungsstrategie einzubauen und ein hinreichend kreatives, dauerhaftes und technisch überzeugendes Paket zu schnüren.

Es überrascht also nicht, dass, auch angesichts eines tendenziell asymmetrischen ,Kampfes ${ }^{\text {‘ }}$ unter den 28 Mitgliedstaaten, Ergebnisse bisweilen ausgeblieben sind. Die Teilung der Lasten für Patrouillen an gemeinschaftlichen Meeresgrenzen, bei Such- und Rettungsmaßnahmen sowie bei der darauffolgenden Aufnahme von Migranten bleibt begrenzt. Die Schiffsbrüchigen vom Herbst 2013, deren Zahl erheblich größer war als die vieler davor, haben die öffentliche Meinung erschüttert und eine momentane Beschleunigung der Verhandlungen zu diesem Thema bewirkt. Im Kern sind bisher jedoch nur marginale Angleichungen in den bereits vorhandenen Dossiers erreicht worden.

Auch in den Beziehungen zu den Ursprungs- und Transitländern, besonders den südlichen Mittelmeer-Anrainern, sind die Ende der 1990er Jahre eingeführten Innovationen (,privilegierte Quoten` für eine begrenzte Anzahl von strategischen Partnern, Koordinationsbemühungen zwischen Migrationspolitik und Entwicklungszusammenarbeit) kontinuierlich zu-

22 Europäische Verteidigungsagentur: Framework Arrangement for Security of Supply between Subscribing Member States, Brüssel.

23 Europäische Kommission: Mitteilung der Kommission an das Europäische Parlament, den Rat, den Europäischen Wirtschafts- und Sozialausschuss und den Ausschuss der Regionen. Ein offenes und sicheres Europa: Praktische Umsetzung, KOM (2014) 154.

24 Das Stockholmer Programm - Ein offenes und sicheres Europa im Dienste und zum Schutz der Bürger, in: Amtsblatt der EU, Nr. C 115 vom 4. Mai 2010, S. 1-38.

25 Grundlegend Jörg Semmler: Das Instrument der Mehrjahresprogramme in der europäischen Innen- und Justizpolitik, in: integration 1/2009, S. 63-74. 
rückgenommen worden. Dies war nicht nur eine Folge der Rezession (Reduzierung der öffentlichen Entwicklungsgelder infolge sinkender Steuereinnahmen), sondern von politischen Richtungswechseln und der damit verbundenen fehlenden Konstanz in der Umsetzung von Politik.

Das Gesamtkonzept für die großen Migrationsthemen in der Europäischen Union muss daher überarbeitet werden. Migration, Asyl und Mobilität sind - trotz ihrer Vielschichtigkeit, auch hinsichtlich der verschiedenen institutionellen Kompetenzen auf europäischer Ebene Politikfelder, die Medien und Politik gegenwärtig mit großer Aufmerksamkeit verfolgen. Reguläre und irreguläre Migration, erzwungene oder spontane Migration sowie Zuströme von außerhalb und Bewegungen innerhalb der Europäischen Union sind Phänomene, die im Laufe der vergangenen Monate einer starken Politisierungswelle unterlagen. Diese hat einen unübersehbaren Widerhall auf der europäischen Agenda erzeugt.

Die Tragödien, die sich im Oktober 2013 in den sizilianischen Gewässern abspielten, haben die Polemik über die Schwächen der derzeitigen Kontroll- und Bergungsaktivitäten an den maritimen Außengrenzen wiederbelebt. Eine wahrgenommene Intensivierung irregulärer Transitmigration innerhalb Europas, auch von Minderjährigen, vom Süden zum Norden des Kontinents hat erneut Streit über das Funktionieren des Schengen-Systems entfacht. Der Schengen-Raum wurde auch zum Gegenstand von Kontroversen in der Union, aufgrund der wiederholten Verschiebung der Entscheidung des Rates der Europäischen Union über die vollständige Anwendung der Bestimmungen des Schengen-Besitzstands auf Rumänien und Bulgarien. ${ }^{26}$ Auch die volle Gewährung der Freizügigkeit in der Europäischen Union war von dem spannungsreichen Klima nicht ausgenommen. Die Regierungen einiger wichtiger Mitgliedstaaten äußerten zunehmend Bedenken, dass dieses elementare Unionsbürgerrecht missbraucht wird und sie haben damit bisweilen scharfe Auseinandersetzungen mit den europäischen Institutionen ausgelöst.

In diesem schwierigen Klima, in dem das gegenseitige Vertrauen auf verschiedenen Ebenen beschädigt erscheint (zwischen den Wählern und EU-Institutionen, zwischen den Mitgliedstaaten, manchmal auch zwischen den EU-Institutionen), müssen die europäischen Institutionen schwierige Koordinationsbemühungen und inter-institutionelle Absprachen anstoßen, um die Prioritäten der Europäischen Union für den Raum der Freiheit, der Sicherheit und des Rechts für die kommenden sieben Jahre festzulegen.

Die italienische Präsidentschaft wird eine entscheidende Rolle in diesem schwierigen Prozess spielen. Der Europäische Rat vom 26.-27. Juni 2014 wird sich zum neuen Arbeitsprogramm in Sachen Recht, Asyl, Sicherheit und Immigration äußern. Es wird für die Präsidentschaft also insbesondere darum gehen, die Maßnahmen, die sich aus den Schlussfolgerungen der Staats- und Regierungschefs ergeben, anzustoßen. Diese Verantwortung kann und muss auch als Chance interpretiert werden. Besonders von einem Land wie Italien, das in verschiedener Hinsicht (als Verantwortlicher für die Kontrolle eines schwierigen Abschnitt der EU-Außengrenze, als Ziel großer Migrationsströme in jüngster Zeit, als bedeutender Ausgangspunkt der EU-Binnenmigration von Jugendlichen) direkt von europäischen Entscheidungen (oder deren Ausbleiben) betroffen ist.

In dieser Phase treffen einige langfristige Interessen Italiens und wichtige strategische Aktionslinien, die von den europäischen Institutionen, und besonders der Kommission ver-

26 Rat der Europäischen Union: Mitteilung an die Presse 3279. Tagung des Rates. Justiz und Inneres. Brüssel, 5. und 6. Dezember 2013, Dok. 17342/13, S. 11. 
fochten werden, in bedeutsamer Weise aufeinander. In Anbetracht dessen, sind folgende Prioritäten hervorzuheben:

Die laufenden Bemühungen für effizientere und nachhaltigere Kontrollen seitens der Europäischen Union und der Mitgliedstaaten an den Außengrenzen - insbesondere denen des Mittelmeers - sollten bei voller Beachtung der Grundrechte weiterverfolgt werden. Ein außerordentlicher und längerfristiger Einsatz (über den momentanen Notstand hinaus) ist nötig, um die Aktionslinien umzusetzen, die von der „Task Force Mediterraneo“ aufgezeigt wurden.

Die langfristigen Maßnahmen mit Ziel der Stärkung des Asylsystems der Europäischen Union sollten fortentwickelt werden. Dies umfasst möglicherweise auch die Revision einiger seiner Bestimmungen, um eine effizientere Anwendung des Solidaritätsprinzips herbeizuführen. Hierbei geht es um eine ausgewogenere Lastenteilung unter den Mitgliedstaaten, besonders bei der Aufnahme gemischter Migrationsströme ${ }^{27}$ und der Aufnahme von Migranten mit dem größten Schutzbedürfnis, wie Minderjährige, Senioren, etc.

Priorität sollte die Weiterführung einer Politik sein, die die Freizügigkeit von Personen innerhalb der Europäischen Union bedingungslos verteidigt und eine Langzeitstrategie verfeinert und verstärkt, die schon von Programmen wie Youth on the Move, Erasmus+ etc. in Gang gesetzt wurde. Ziel ist es, die positiven Auswirkungen der Mobilität, besonders von Jugendlichen, zu maximieren. Wirtschaftliche und kulturelle Dynamiken müssen dabei in Betracht gezogen werden, um mögliche negative Auswirkungen zu verhindern.

Aus einem breiteren Blickwinkel betrachtet sind die Verknüpfungen von Migrations- und Mobilitätspolitik, Sozial- und Integrationspolitik sowie verstärkte europäische Antworten auf die soziale Dimension der Krise von essenzieller Bedeutung. Dies ist vor dem Hintergrund der stark asymmetrischen sozialen Auswirkungen der Krise zu sehen, die zu Mobilitätsprozessen unter schwierigen Bedingungen führen und Risiken für soziale und politische Spannungen bergen. In diesem Zusammenhang erscheint es auch notwendig, europäische Maßnahmen gegen Rassismus und Diskriminierung wiederzubeleben. Die Erklärung von Rom vom September 2013 liefert einen ersten Impuls für ein Abkommen zu einem Europa der Vielfalt und des Kampfes gegen Rassismus (2014-2020).

\section{Die strategischen Linien Italiens für die institutionelle Komponente der EU: mehr Effizienz und demokratische Legitimation}

Institutionelle Reformen der Europäischen Union standen in den letzten Jahren im Mittelpunkt der Debatte und es folgten daraus konkrete Initiativen. Die Fortschritte und Reformen der sogenannten wirtschaftspolitischen Steuerung waren bei Weitem schneller und tiefgreifender als in den Jahrzehnten nach der Unterzeichnung des Vertrags von Maastricht. Auf der Ebene des Europäischen Rates wurde in den vergangenen drei Jahren fast ausschließlich daran gearbeitet, Mechanismen, Verfahren und Institutionen zu entwerfen, um die Defizite der Wirtschafts- und Währungsunion auszugleichen.

Was Italien betrifft, ist zu erwähnen, dass die Zukunft Europas viel mehr als in der Vergangenheit zu einem wichtigen, aber auch spaltenden Element in der Innenpolitik geworden ist. Somit wird dieses Thema unmittelbar Einfluss auf das innenpolitische Machtverhältnis haben, beginnend mit den Wahlen zum Europäischen Parlament. Daher müssen die pro-

27 Als gemischte Migrationsströme werden solche bezeichnet, die sowohl Asylsuchende als auch Wirtschaftsflüchtlinge umfassen. 
europäischen Kräfte die Karte der institutionellen Zukunft Europas mit äußerster Vorsicht spielen. Andernfalls laufen sie Gefahr, zu einem Zeitpunkt einer unkritischen ideologischen Haltung beschuldigt zu werden, zu dem die nationale öffentliche Meinung dazu tendiert, die Weiterentwicklung der Europäischen Union (also auch die des Euros) unmittelbar mit der Fortdauer der gegenwärtigen Rezession ohne Wachstumsperspektiven in Verbindung zu bringen.

Abgesehen von diesen Überlegungen zum negativen politischen Klima, das die Zukunft der politischen Institutionen der Europäischen Union umgibt, gilt es dennoch, die italienischen Prioritäten auf dem schwierigen Terrain institutioneller Reformen der Union zu benennen und eine Strategie für deren Erreichen in Etappen festzulegen.

Bei der ersten Etappe geht es darum, die Vollendung der in den letzten Jahren angestoßenen Initiativen zu fördern und diese noch genauer zu definieren. Wie bereits angedeutet, konzentriert sich Italien insbesondere auf die Vollendung der Bankenunion: der Start des Überwachungsmechanismus für die Gruppe der größeren Banken seitens der Europäischen Zentralbank; die Annahme einheitlicher Kriterien für die Lösung von Bankenkrisen; die Schaffung einer glaubhaften europäischen Einlagensicherung; und schließlich die Einleitung eines effizienten einheitlichen Mechanismus zur Bankenabwicklung. Es ist im Interesse eines jeden Landes, dass diese Instrumente funktionieren und in kürzest-möglicher Zeit vervollständigt werden. In jedem Fall sollte uns, insbesondere hinsichtlich des einheitlichen Mechanismus zur Bankenabwicklung, weniger beschäftigen, ab wann genau dieser funktionsfähig ist, als vielmehr die Glaubwürdigkeit und Effizienz des entsprechenden Entscheidungsmechanismus. Dies sind kleine Schritte auf dem Weg zu einer effizienteren Regulierung der Wirtschaft, die jedoch für die zukünftige Glaubwürdigkeit des Euros und der Europäischen Union äußerst wichtig sind.

Die zweite Strategie bezieht sich auf die sogenannten vertraglichen Vereinbarungen, die besonders im Laufe der halbjährigen Präsidentschaft Italiens definiert werden sollen. Auch hier wird es nötig sein, die Natur des Instrumentes korrekt zu bestimmen und dessen Anwendung in allen Ländern der Eurozone sicherzustellen sowie glaubhafte und effiziente Solidaritätsziele zu benennen. Die Entscheidungsmechanismen sollten dann so einfach wie möglich sein, um ihre Anwendung unmittelbar und transparent zu gestalten. Angesichts der öffentlichen Meinung, die der bislang betriebenen Sparpolitik der Europäischen Union äußerst kritisch gegenüber steht, ist es wichtig, positive Nachrichten präsentieren zu können.

An dritter Stelle sollte einem mittelfristigen Aspekt große Aufmerksamkeit zukommen: dem Europäischen Fiskalpakt und seiner vorgesehenen Integration ${ }^{28}$ in das europäische Primärrecht. Dieses Vorhaben ist in mehrfacher Hinsicht besonders schwierig. Die erste Herausforderung ist es, einen internationalen Vertrag zu ändern, der 26 europäische Staaten in ein System der verstärkten Zusammenarbeit innerhalb des institutionellen Rahmens der Europäischen Union einbindet. Auf diese Weise werden Doppeldeutigkeiten, die sich aus der Verknüpfung von Verträgen unterschiedlicher rechtlicher Natur ergeben, beseitigt. Das zweite bedenkenswerte Element ist, dass die Integration des Fiskalpakts in den institutionellen Rahmen der europäischen Verträge mittelfristig auch eine Gelegenheit bietet, die anderen ,außer-institutionellen“ Instrumente der Krisenbewältigung (beispielsweise den Europäischen Stabilitätsmechanismus) in die EU-Verträge zu integrieren. Es handelt sich um eine notwendige Operation zur Vereinfachung der verschiedenen institutionellen Initiativen,

28 Art. 16 Fiskalvertrag. 
die in den vergangenen Krisenjahren verabschiedet wurden. Eine partielle Revision des Lissabon-Vertrags muss daher auch in Aussicht gestellt werden.

Schließlich gilt es zu beachten, dass sich für die Zukunft der Europäischen Union Risiken wie Chancen aus dem Umstand ergeben, dass sich die Union mit dem britischen Wunsch nach einer Neuverhandlung der Konditionen und Modalitäten der künftigen Teilnahme Großbritanniens an der Europäischen Union auseinander setzen muss. Es ist noch zu früh, die Konsequenzen eines solchen Szenarios zu bewerten, da die möglichen Forderungen Londons noch unklar sind. Eine derartige Perspektive könnte jedoch auch eine Gelegenheit für den Entwurf eines umfassenderen und radikaleren Konzepts zur Überarbeitung der Zielsetzung der Europäischen Union und der dazugehörigen Institutionen bieten. Diese Frage stellt sich selbstverständlich erst mittelfristig, aber sollte bereits heute angemessen in Überlegungen zur Zukunft der Europäischen Union einbezogen werden. ${ }^{29}$

Letztlich geht es darum, beginnend mit der Kampagne für die Europawahlen und dem Halbjahr der italienischen Präsidentschaft, erste Überlegungen zu möglichen Anpassungen und institutionellen Reformen der zukünftigen Union zu skizzieren. Schritte zur Vervollständigung der entworfenen Instrumente und Politiken für eine bessere europäische wirtschaftspolitische Steuerung sind hierbei von zentraler Bedeutung. Dazu gehören die notwendige Vereinfachung und ,Vergemeinschaftung', die sukzessive Einbindung in den institutionellen Rahmen des europäischen Primärrechts und schließlich der allmähliche Beginn einer Vertragsreform im Zuge eines britischen Antrags zur Neuverhandlung seiner EU-Mitgliedschaft. Das übergeordnete Ziel aller Bemühungen ist es, im gemeinschaftlichen Sinne ein neues institutionelles Gleichgewicht herzustellen, das derzeit sowohl den Institutionen also auch den Mitgliedstaaten entglitten ist.

\section{Umfassende Allianzen und ein neuer Ton in der Wirtschaftspolitik}

Die Gesamtheit der Ziele der italienischen Ratspräsidentschaft, die auf diesen Seiten kurz skizziert wurden, ist nur dann sinnvoll und realistisch, wenn es gelingt, hierfür Allianzen von Mitgliedstaaten zu bilden, die über das Halbjahr hinaus reichen und die mittel- und langfristige Perspektiven mit einbeziehen. Hierbei handelt es sich um das für jede Gemeinschaftsaktivität übliche Bilden von Koalitionen. Bei den Themen der Migrationspolitik wäre es beispielsweise nötig, eine erste Konsenslinie unter den Staaten zu ziehen, die die meisten Migranten aufnehmen. Dies wird jedoch sicher nicht genügen, sofern die größeren ,Spieler ${ }^{6}$ im Zentrum und im Norden Europas nicht auch in die Formulierung der Migrationspolitiken der Europäischen Union einbezogen werden. Das Gleiche gilt für die anderen dargelegten Prioritäten Italiens. Ganz oben steht die Kooperation im Bereich der Finanz- und Wirtschaftspolitik.

Niemand in Italien ist so naiv zu glauben, dass politische Maßnahmen für Wachstum und produktive Investitionen ohne die Unterstützung Deutschlands verabschiedet werden könnten. Im Laufe der vergangenen Jahre haben sich in der italienischen Öffentlichkeit und in der Opposition des Landes immer wieder Stimmen gegen die von Deutschland so stark propagierte ,Philosophie der Regeln und Zahlen` erhoben. Dennoch hat keine italienische Regierung - von Mario Monti über Enrico Letta bis hin zur aktuellen von Matteo Renzi - je angezweifelt, dass Stabilität der Ausgangspunkt für die neuen Wirtschaftspolitiken der Eu-

29 Als Debattenbeitrag zur Zukunft der Europäischen Union siehe Andrew Duff: Zurück zur Kernfrage: eine föderale Ordnung für Europa, in: integration 1/2014, S. 65-71. 
ropäischen Union ist. Nach Jahren der Rezession und des mageren Wachstums müssen sich die Nachrichten aus Brüssel (und aus Berlin) jedoch im Ton ändern. Dies dient nicht nur dem Ziel, den Euroskeptikern (in Italien und andernorts) keine weiteren Argumente an die Hand zu geben. Es geht vor allem darum, die europäische Wirtschaft wieder in Schwung zu bringen, die sich in ihrer Gesamtheit global gesehen im Niedergang befindet. Die Beziehungen zwischen Rom und Berlin werden zweifellos stabil bleiben, aber die Tonlage muss sich ändern.

\section{Europa in der Globalisierung der „langen“ 1970er Jahre}

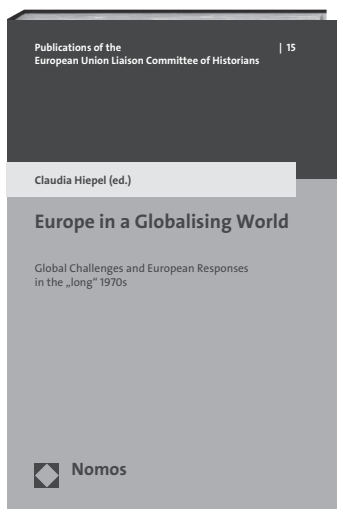

\author{
Europe in a Globalising World \\ Global Challenges and European Responses \\ in the „long“ 1970s \\ Herausgegeben von Claudia Hiepel \\ 2014, 374 S., brosch., 68,- $€$ \\ ISBN 978-3-8487-1343-1 \\ (Veröffentlichungen der Historiker-Verbindungs- \\ gruppe bei der Kommission der EG, Bd. 15) \\ www.nomos-shop.de/22928
}

Der Globalisierungsschub der 1970er Jahren hat nicht zu einem Bedeutungsverlust der Europäischen Gemeinschaft geführt, vielmehr ging er mit einer Europäisierung Europas einher. Mit dem Ausbau von Handlungsfeldern und Interaktionsräumen hat die Gemeinschaft auf die Globalisierung reagiert und die regionale Integration in Europa forciert.

Portofreie Buch-Bestellungen unter www.nomos-shop.de

Alle Preise inkl. Mehrwertsteuer 